\title{
Atendimento de emergência às vítimas de acidentes e violências no Brasil
}

\author{
Suely Ferreira Deslandes ${ }^{1}$, Maria Cecília de Souza Minayo ${ }^{2}$ \\ e Maria Luiza Carvalho de Lima $^{3}$
}

Como citar Deslandes SF, Minayo MCS, Lima MLC. Atendimento de emergência às vítimas de acidentes e violências no Brasil. Rev Panam Salud Publica. 2008;24(6):430-40.

RESUMO Objetivo. Analisar os êxitos, lacunas, problemas e potencialidades da aplicação da Politica Nacional de Redução de Morbimortalidade por Acidentes e Violências (PNRMAV) no Brasil quanto à atenção hospitalar de emergência e urgência.

Métodos. De 2004 a 2007, uma pesquisa foi realizada em quatro capitais e no Distrito Federal. Os seguintes aspectos foram investigados: existência de padronização de condutas de atendimento às vítimas de acidentes e violências; oferta e adequação de leitos de terapia intensiva (adulta e pediátrica); capacitação dos profissionais para o atendimento; existência de articulação intersetorial para melhor atendimento às vítimas; e estruturação e organização dos serviços de atendimento hospitalar. A operacionalização incluiu uma fase exploratória, trabalho de campo, análise e divulgação dos dados. Para a análise, foram utilizados métodos quantitativos (freqüências simples e relativas) e qualitativos (análise de interações entre conteúdos manifestos e inferências de conteúdos latentes nos depoimentos e anotações de campo).

Resultados. O estudo revelou resultados positivos associados à implantação da PNRMAV e permitiu identificar os seguintes pontos de atenção: 1) necessidade de investimento na resolutividade dos serviços de atenção básica, 2) importância de se fortalecer e articular a rede de urgência e emergência, 3) relevância dos investimentos em equipamentos adequados e sua manutenção no ritmo exigido pelos atendimentos e 4) necessidade de investimentos na formação permanente de profissionais.

Conclusões. Os sucessos parciais alcançados pela implantação da PNRMAV mostram que é possível incluir o tema da violência e dos acidentes na pauta da saúde pública e atuar com estratégias em diferentes níveis a favor da vida.

Palavras-chave Acidentes, medicina de emergência, políticas públicas de saúde, violência, Brasil.

No Brasil, as violências e os acidentes constituem, desde o início da dé-

\footnotetext{
1 Fundação Oswaldo Cruz, Instituto Fernandes Figueira. Enviar correspondência para Suely Ferreira Deslandes no seguinte endereço: Avenida Rui Barbosa 716, segundo andar, Flamengo, CEP 22250-020, Rio de Janeiro, RJ, Brasil. Fone: +55-212554.1796; fax: +55-21-2552.8898; e-mail: desland@ iff.fiocruz.br

2 Fundação Oswaldo Cruz, Escola Nacional de Saúde Pública, Centro Latino-Americano de Estudos de Violência e Saúde (CLAVES). E-mail: cecilia@claves.fiocruz.br

3 Fundação Oswaldo Cruz, Centro de Pesquisas Aggeu Magalhães. E-mail: luiza@cpqam.fiocruz.br
}

cada de 1980, a segunda causa mais freqüente de mortalidade no país como um todo e a primeira causa para a ampla faixa etária de 5 a 49 anos. Embora as estatísticas dos últimos 3 anos indiquem uma lenta redução nas taxas de homicídios e acidentes de trânsito (as duas principais causas violentas de óbito no Brasil), as causas externas apresentam-se ainda como a sexta causa de internações e constituem uma significativa de- manda aos serviços de urgência e emergência (1).

Com o impacto dos acidentes e violências na demanda assistencial das urgências e emergências dos hospitais brasileiros, principalmente nos grandes centros urbanos, é preciso considerar a necessidade de estruturação de uma rede assistencial hierarquizada e resolutiva. A pressão sobre os serviços de atendimento se acentua ainda mais com a constante invasão de pacientes 
portadores de agravos que poderiam encontrar resolução nos serviços da rede básica e nas unidades de urgência de baixa complexidade (2). Sabe-se, entretanto, que investimentos maciços na prevenção dos acidentes e violências e na promoção de ambientes nãoviolentos poderiam fazer diminuir com mais efetividade a pressão da demanda sobre os serviços hospitalares de urgência e de reabilitação.

Em 2001, publicou-se no Brasil a Política Nacional de Redução da Morbimortalidade por Acidentes e Violências (PNRMAV) $(3,4)$, cuja relevância, dentre outros aspectos, deriva do fato de que ela faz uma inflexão e uma ampliação da responsabilidade do setor de saúde sobre o tema das violências, historicamente restrito aos foros policiais e judiciários. A partir dessa política, o Ministério da Saúde brasileiro produziu várias portarias específicas, criando diretrizes e orientações sobre a promoção da vida e a prevenção da violência, além de orientar o Sistema de Atenção à Saúde quanto aos atendimentos às vítimas e seus familiares. $\mathrm{A}$ PNRMAV constitui, atualmente, o principal parâmetro de análise e avaliação das respostas do Sistema Único de Saúde (SUS) às complexas demandas criadas pelas violências e acidentes (5).

No decorrer dos últimos 20 anos, essas demandas motivaram ainda a elaboração de portarias pelo Ministério da Saúde com o objetivo de difundir os conceitos, as diretrizes e as ações nessa área $(6,7)$. Por exemplo, em 2002, foi instituído o Regulamento Técnico dos Sistemas Estaduais de Urgência e Emergência, como resultado da necessidade de ordenar o acesso ao atendimento de urgências e emergências, com garantia de acolhimento, a primeira atenção qualificada e resolutiva para as urgências de baixa e média complexidade, a estabilização, quando necessária, e a referência adequada dos pacientes graves dentro do SUS (8). O regulamento discorre sobre as atribuições, o dimensionamento e a organização estrutural dos sistemas, bem como sobre aspectos da interação com os demais serviços e emergências em seus vários níveis de complexi- dade, incluindo assim as emergências e urgências dos hospitais gerais e de referência. Há um detalhamento sobre recursos humanos, sobre as devidas habilitações e sobre a capacitação para o trabalho nessas unidades. Há, ainda, considerações sobre área física, material de consumo, medicamentos, equipamentos e estrutura da grade de referência que dá suporte a esses serviços, a saber: regulação médica de urgência, atendimento pré-hospitalar fixo e móvel, transporte inter-hospitalar e criação de núcleos de urgência com a proposição de grades curriculares para a capacitação de recursos humanos da área (8).

Nesse sentido, a atenção hospitalar às vítimas de acidentes e violências reúne de forma complexa a estrutura física, a disponibilidade de insumos, $\mathrm{o}$ aporte tecnológico e os recursos humanos especializados para intervir nas situações de emergência decorrentes dos acidentes e violências. As emergências são a principal porta de entrada desses pacientes no hospital; considerando a gravidade das lesões, a assistência demandará ações de diferentes serviços e poderá exigir um tempo considerável de internação, acarretando um custo elevado $(5,9)$.

Diante desse quadro, o objetivo do presente artigo foi analisar os êxitos, as lacunas, os problemas e as potencialidades da aplicação da PNRMAV (4) no que concerne à atenção hospitalar de urgência e emergência (6). Este artigo apresenta resultados selecionados de uma pesquisa mais ampla, publicada sob o formato de relatório, na qual foi feita uma análise diagnóstica de todo o sistema (pré-hospitalar, hospitalar, de reabilitação e de formação de recursos humanos) para o atendimento de acidentes e violências.

\section{MÉTODO}

A pesquisa que deu origem a este artigo (5) foi realizada em quatro capitais brasileiras e no Distrito Federal. Em 2007, as populações estimadas das cinco localidades eram as seguintes: Rio de Janeiro, 6093472 habitantes; Recife, 1533580 habitantes; Manaus,
1646602 habitantes; Curitiba, 1797408 habitantes; e Distrito Federal, 2455903 habitantes.

O estudo ocorreu no período de 2004 a 2007 e resultou da cooperação entre o Centro Latino-Americano de Estudos de Violência e Saúde (CLAVES) da Fundação Oswaldo Cruz e vários centros de pesquisa das localidades estudadas: Departamento de Serviço Social da Universidade de Brasília, Núcleo de Estudos e Saúde Coletiva da Universidade Federal do Rio de Janeiro, Faculdade de Educação da Universidade Federal do Amazonas, Pontifícia Universidade Católica do Paraná e Faculdade de Ciências Médicas da Universidade de Pernambuco.

\section{Seleção das cidades estudadas}

A escolha das cidades foi feita a partir de um estudo que ordenou por magnitude os 224 municípios brasileiros com população acima de 100000 habitantes (10). Foi criado um índice sintético de violência que considerou o número de óbitos e as taxas de mortalidade por homicídio, acidente de transporte e suicídio no ano de 2000. Segundo esse índice, o Rio de Janeiro situou-se em segundo lugar no país, Recife em terceiro, Brasília em quinto, Manaus em $13^{\circ}$ e Curitiba em $17^{\circ}$. Com exceção do Rio de Janeiro, todas as localidades foram as primeiras colocadas quanto a taxas de violência a partir do indicador composto em suas respectivas regiões, sendo esse o critério definidor da escolha.

No que se refere à morbidade, constatou-se que, naquele ano de 2000, as cinco cidades somaram 45434 internações por causas externas, representando 6,5\% das hospitalizações no país (10). No caso de Brasília, optou-se por analisar o Distrito Federal e a rede de atendimento das localidades que compõem o seu entorno (Gama, Taguatinga, Brazilândia, Sobradinho, Planaltina, Paranoá, Núcleo Bandeirante, Ceilândia, Guará, Samambaia, Santa Maria, São Sebastião, Recanto das Emas, Riacho Fundo e Candangolândia). 


\section{Operacionalização e instrumentos utilizados para a coleta e análise dos dados}

O conceito metodológico básico deste estudo é o de análise diagnóstica. Essa forma de análise compreensiva e interpretativa da realidade busca, por um lado, dar conta dos êxitos e das dificuldades do sistema de saúde em atender a crescente demanda e as mudanças qualitativas das lesões e traumas provenientes das violências e acidentes, e por outro, construir alternativas e possibilidades para o melhor desenvolvimento da gestão. Assim, o termo diagnóstico configura uma análise situacional que leva em conta as condições gerais de demanda à luz da infra-estrutura de equipes e serviços existentes.

Do ponto de vista operacional, seguimos os princípios da triangulação, estratégia que visa à combinação e ao cruzamento de múltiplos pontos de vista, técnicas, estratégias e métodos (11-13). O referencial principal da pesquisa e de todas as variáveis de análise foi o próprio documento da PNRMAV (4) em seu marco lógico (finalidade, propósitos, componentes e atividades) no que concerne ao atendimento hospitalar. Os documentos de apoio foram: a portaria 1.863/GM (14), que institui a Política Nacional de Atenção às Urgências; a portaria SVS 466 (15), que regulamenta o funcionamento de serviços de tratamento intensivo; a portaria 479/GM (16), que cria mecanismos para a implantação dos sistemas estaduais de referência hospitalar em atendimento de urgências e emergências; e a portaria 2.048/GM (8), que aprova o regulamento técnico dos Sistemas Estaduais de Urgência e Emergência.

A partir desses marcos operadores, foram elaborados indicadores para a análise dos serviços. Os indicadores foram validados por especialistas através da técnica Delphi (5), abordando os seguintes aspectos: existência de normas de atendimento às vítimas de acidentes e violências para padronização de condutas, racionalização do atendimento e redução de custos; oferta e adequação de leitos de terapia inten- siva (adulta e pediátrica); capacitação dos profissionais para o atendimento; existência de articulação intersetorial para melhor atendimento às vítimas; $\mathrm{e}$ estruturação e organização dos serviços de atendimento hospitalar.

Os indicadores formulados para analisar os serviços foram: 1) proporção de unidades que possuem rotinas/protocolos de atendimento às vítimas de acidentes e violências acessíveis aos profissionais; 2) proporção de unidades que possuem unidade de tratamento intensivo (UTI); 3) proporção de unidades com UTI adequada aos requisitos básicos; 4) proporção de hospitais para o atendimento às vítimas de acidentes e violências com número mínimo de profissionais indispensáveis para seu funcionamento; 5) proporção de hospitais com recursos tecnológicos (mínimos e indispensáveis) adequados e suficientes; 6) taxa de mortalidade hospitalar da emergência; 7) proporção de unidades com mecanismos próprios ou articulados com outros serviços para a realização de transferência e transporte de pacientes; 8) proporção de unidades com mecanismos próprios ou articulados com outros serviços para a realização de suporte ininterrupto de laboratório de radiologia e patologia clínica; 9) proporção de hospitais que dispõem de protocolos e rotinas acessíveis aos profissionais para atenção às vítimas de acidentes e violências.

Além dos indicadores citados, avaliamos dois aspectos referentes à organização hospitalar: articulação com a rede hospitalar via central de regulação ou rede pactuada e oferecimento de reabilitação, suporte e acompanhamento às vítimas de violência e seus familiares, aos agentes da agressão e aos profissionais que atendem as vítimas de violência.

A operacionalização da investigação foi construída em torno de quatro fases de trabalho (fase exploratória, trabalho de campo, análise e divulgação dos dados), cada qual agregando diferentes ciclos de pesquisa. A primeira fase envolveu a consulta a especialistas e gestores membros dos três subsistemas em estudo (pré- hospitalar, hospitalar e de reabilitação), tendo sido feitas 23 entrevistas semi-estruturadas. A análise dessa etapa subsidiou a construção de instrumentos para o melhor conhecimento do subsistema hospitalar.

Na segunda fase, foi feito o mapeamento da rede de atendimento às vítimas de acidentes e violências. $\mathrm{O}$ instrumento aplicado em cada unidade de saúde que realizava tal atendimento incluía três blocos de variáveis: identificação; caracterização das atividades de atendimento; e descrição da estrutura existente. Na terceira fase, os serviços foram novamente visitados, sendo aplicados novos questionários, agora destinados a analisar o tipo de atendimento prestado às vítimas de acidentes e violências. Os questionários foram estruturados em dois blocos de questões, o primeiro caracterizando a estrutura existente (27 questões) e o segundo, a organização do serviço (22 questões).

Os instrumentos foram aplicados ao gerente de cada unidade ou a um profissional delegado por ele como o mais indicado a prestar tais informações. Foram incluídos todos os serviços que atendiam vítimas de violências nas referidas cidades, totalizando um acervo de 53 hospitais (cinco em Curitiba, sete em Manaus, 10 em Recife, 15 no Distrito Federal e 16 no Rio de Janeiro). Foram aplicadas 10 novas entrevistas semi-estruturadas com os gestores, buscando captar sua ótica sobre a situação de cada cidade em relação ao atendimento das vítimas de acidentes e violências, ao cumprimento das diretrizes da PNRMAV e aos demais parâmetros de qualidade de atendimento preconizados nas políticas e portarias mais importantes de cada um desses três setores de atenção.

A quarta fase da pesquisa foi dedicada à análise crítica e à melhoria da qualidade dos dados e da validade das interpretações produzidas passo a passo. Nesse movimento, cada relatório local foi analisado por profissionais de estatística, epidemiologia e ciências sociais do CLAVES e reenviado às equipes colaboradoras para que ajustassem as informações e a forma de apresentação das mesmas. A partir 
daí, os relatórios finais das cidades serviram de base para a construção de uma análise transversal.

Os questionários foram digitados em um banco de dados (EpiData) (17) e criticados. A análise teve um caráter exploratório, sendo usadas medidas estatísticas descritivas (freqüências simples e relativas) aplicadas às variáveis em cada cidade. A análise qualitativa buscou interações entre conteúdos manifestos e inferências de conteúdos latentes nos depoimentos e anotações de campo. As entrevistas foram processadas a partir de leitura temática e, a seguir, os trechos pertinentes foram codificados, identificando argumentos, significados e relatos de práticas relevantes aos objetivos do trabalho. A seguir foi realizado um exercício de triangulação a partir de dados objetivos, da análise de relações e representações dos diferentes atores, buscando uma compreensão integrada do processo.

Para produzir este artigo, foram utilizados os dados primários da pesquisa para mostrar a análise situacional do atendimento de emergência. Embora sem possibilidade de extrapolação - tanto em relação aos êxitos como em relação aos problemas -, este artigo oferece informações sobre questões comuns e reincidentes em toda a rede, sobretudo nas capitais e regiões metropolitanas, que concentram a maior parte dos acidentes e violências no país (10).

\section{RESULTADOS}

\section{Existência e adequação das unidades de tratamento intensivo}

Os indicadores "proporção de hospitais que possuem UTI" e "adequação das unidades aos parâmetros estabelecidos pelo Ministério da Saúde" foram utilizados para a avaliação da atenção hospitalar às vítimas de acidentes e violências. A existência de UTI em todos os hospitais secundários ou terciários com capacidade igual ou superior a 100 leitos é obrigatória. Os leitos de UTI devem corresponder a 5 a $10 \%$ do total de leitos existentes no hospital (variando o percentual de acordo com o porte e a complexidade da unidade), devem ter atendimento 24 horas, área física própria e acesso facilitado. Da mesma forma, $5 \%$ do total de leitos pediátricos devem ser destinados à UTI (18). Em Manaus e em Curitiba, 100\% dos hospitais possuíam UTI; em Recife, 80\%; no Rio de Janeiro, 87,5\%; e no Distrito Federal, $60 \%$.

Em relação à adequação das UTI segundo os requisitos recomendados (equipamentos e estrutura física), nenhuma das cidades apresentou resultado satisfatório. Recife alcançou a proporção mais elevada, com $50 \%$ dos hospitais adequados. Em Manaus, no Rio de Janeiro e em Curitiba, 14, 19 e $20 \%$ dos hospitais, respectivamente, estavam de acordo com as normas estabelecidas. No Distrito Federal, nenhum dos hospitais que atendem vítimas de acidentes e violências possuíam UTI em conformidade com os requisitos básicos, o que surpreende negativamente diante da morbimortalidade existente.

No detalhamento das condições de estrutura e equipamentos, observou-se que os sete hospitais analisados em Manaus possuíam UTI e que quatro também contavam com unidades de tratamento semi-intensivo (UTSI). Nos sete hospitais com UTI, esta oferecia atendimento 24 horas e apresentava área física própria, com acesso facilitado. Cinco unidades possuíam UTI de alta complexidade (tipo C) e duas unidades possuíam UTI de complexidade intermediária (tipo B).

Em Recife, das 10 unidades analisadas, oito possuíam UTI e nove tinham UTSI. Em nove hospitais, as UTI e UTSI ofereciam atendimento 24 horas e acesso facilitado à área física. Oito hospitais possuíam UTI tipo $\mathrm{C}$, nove UTI tipo B e quatro UTI tipo A (menor complexidade).

No Distrito Federal, dos 15 hospitais analisados, nove possuíam UTI e seis possuíam UTSI. Nove hospitais contavam com atendimento 24 horas e 10 apresentavam área física própria com acesso facilitado à UTI. Seis hospitais possuíam UTI tipo B e seis UTI tipo A. Não havia UTI tipo $C$ disponível na rede hospitalar do SUS para atenção às vítimas de acidentes e violências no Distrito Federal, o que confirma o resultado de $0 \%$ para o indicador referente à adequação das UTI, mencionado anteriormente.

No Rio de Janeiro, dos 16 hospitais analisados, 14 possuíam UTI e nove possuíam UTSI. Nos primeiros 14 , as UTI realizavam atendimento 24 horas e apresentavam área física própria. No entanto, apenas 13 tinham acesso físico facilitado à UTI. Dez hospitais tinham UTI tipo C, oito tipo B, e cinco tipo A.

Em Curitiba, os cinco hospitais possuíam UTI e quatro tinham UTSI atendendo 24 horas, em área física própria e com acesso facilitado a ambulâncias. Dois hospitais contavam com UTI tipo C, dois com UTI tipo B, e um com UTI tipo A.

Analisando o número de leitos ofertados por especialidade nos hospitais da rede de atenção às vítimas de acidentes e violências nas cidades estudadas (tabela 1), observa-se um déficit de leitos de emergência e de UTI. No Rio de Janeiro, seis hospitais não possuíam leitos de UTI pediátrica, sendo que o número total desse tipo de leito no município se iguala ao número encontrado no Distrito Federal, onde a população é quase três vezes menor. Em Curitiba, apenas um hospital oferecia leitos de UTI de retaguarda.

\section{Recursos tecnológicos e humanos}

Outro indicador analisado para avaliar a estrutura foi a "disponibilidade de recursos tecnológicos existentes e sua adequação e funcionamento". $\mathrm{Na}$ maioria das cidades estudadas, esses recursos tecnológicos existiam e, em grande parte, funcionavam de forma adequada. No entanto, sua quantidade não foi considerada suficiente pelos gestores para atender às necessidades da população assistida (tabela 2). Por exemplo, nenhum dos hospitais investigados possuía banco de tecidos ou intensificador de imagens, e o serviço de tomografia era oferecido em apenas cinco hospitais. Recursos como neurocirurgia, cirurgia cardiovascular e cirurgia pediátrica, freqüentemente necessários no atendimento às vítimas de 
TABELA 1. Número de leitos nos hospitais que atendem pessoas em situação de acidentes e violências nas capitais, Brasil, 2006 ${ }^{\mathrm{a}}$

\begin{tabular}{lcrccc}
\hline Leitos hospitalares & Manaus & Recife & Distrito Federal & Rio de Janeiro & Curitiba \\
\hline Total & 949 & 1692 & 1222 & 4181 & 1758 \\
Urgência/emergência & 246 & 455 & 261 & 837 & 447 \\
Adultos & 645 & 1591 & 731 & 1877 & 933 \\
Pediátricos & 254 & 378 & 343 & 375 & 529 \\
UTI adulto & 53 & 64 & 64 & 146 & 60 \\
UTI pediátrica & 25 & 34 & 73 & 73 & 109 \\
UTI de retaguarda & 5 & 67 & 21 & 33 & 10 \\
\hline
\end{tabular}

a $O$ total de leitos não corresponde ao somatório das categorias, uma vez que estas não são excludentes.

violências e acidentes, estavam disponíveis em apenas quatro hospitais, nem sempre em condições adequadas.

Em Recife, os recursos tecnológicos para análise laboratorial, eletrocardiografia, radiologia convencional, endoscopia, ultra-sonografia, banco de sangue, anestesiologia, ecocardiografia e cirurgia cardiovascular estavam presentes nos 10 serviços hospitalares analisados. Contudo, apenas três unidades possuíam banco de tecidos.

A princípio, a rede hospitalar local deveria estar estruturada e organizada para atender todas as demandas decorrentes das situações de acidentes e violências. No entanto, em Recife, conforme observado, poucos serviços possuíam esses recursos de forma suficiente e adequada. Além disso, apenas dois serviços de eletrocardiografia e dois de anestesiologia foram classificados como suficientes; nos demais casos, tal questão não foi respondida.

Também no Distrito Federal os hospitais dispunham dos recursos tecnológicos necessários com um grau de adequação razoável, porém insuficiente para o atendimento da demanda. A angiografia foi o único procedimento não realizado em nenhum dos 15 hospitais analisados. Em 11 hospitais havia eletrocardiograma e banco de sangue. Esses recursos foram avaliados como adequados em 10 e sete serviços, respectivamente, mas foram considerados suficientes em apenas quatro hospitais.

TABELA 2. Distribuição dos serviços hospitalares segundo a disponibilidade, adequação e suficiência de recursos tecnológicos, Brasil, 2006

\begin{tabular}{|c|c|c|c|c|c|c|c|c|c|c|c|c|c|c|c|}
\hline \multirow{2}{*}{$\begin{array}{l}\text { Recurso } \\
\text { tecnológico para }\end{array}$} & \multicolumn{3}{|c|}{$\begin{array}{c}\text { Manaus } \\
(7 \text { hospitais })^{\mathrm{a}}\end{array}$} & \multicolumn{3}{|c|}{$\begin{array}{c}\text { Recife } \\
(10 \text { hospitais })^{\mathrm{a}}\end{array}$} & \multicolumn{3}{|c|}{ 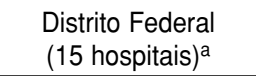 } & \multicolumn{3}{|c|}{ 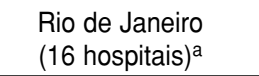 } & \multicolumn{3}{|c|}{$\begin{array}{c}\text { Curitiba } \\
(5 \text { hospitais })^{\mathrm{a}}\end{array}$} \\
\hline & $\mathrm{D}$ & A & $S$ & D & A & $S$ & $\mathrm{D}$ & A & $S$ & $\mathrm{D}$ & $A$ & S & $\mathrm{D}$ & A & S \\
\hline \multicolumn{16}{|l|}{ Análise clínica/ } \\
\hline laboratorial & 7 & 6 & 4 & 10 & 2 & - & 14 & 11 & 5 & 16 & 13 & 8 & 5 & 4 & 4 \\
\hline Eletrocardiografia & 6 & 6 & 4 & 10 & 5 & 2 & 11 & 10 & 4 & 16 & 14 & 10 & 5 & 5 & 5 \\
\hline Radiologia & 7 & 7 & 6 & 10 & 5 & - & 12 & 9 & 4 & 16 & 14 & 11 & 5 & 5 & 5 \\
\hline Banco de sangue & 7 & 5 & 4 & 10 & 5 & - & 11 & 7 & 4 & 13 & 12 & 4 & 5 & 5 & 5 \\
\hline Banco de tecidos & - & 一 & - & 3 & - & - & 1 & 1 & 1 & 1 & 1 & 1 & 2 & 2 & 2 \\
\hline Broncoscopia & 4 & 3 & 1 & 9 & 3 & - & 5 & 4 & 1 & 6 & 6 & 5 & 5 & 4 & 4 \\
\hline Tomografia & 5 & 2 & 2 & 9 & 3 & - & 3 & 2 & 2 & 12 & 8 & 5 & 5 & 4 & 4 \\
\hline Anestesiologia & 7 & 5 & 5 & 10 & 3 & 2 & 9 & 6 & 2 & 15 & 13 & 14 & 5 & 4 & 4 \\
\hline \multicolumn{16}{|l|}{ Terapia renal } \\
\hline Angiografia & 2 & 1 & 1 & 10 & 2 & - & - & - & - & 3 & 3 & 2 & 3 & 2 & 2 \\
\hline \multicolumn{16}{|l|}{ Cirurgia } \\
\hline cardiovascular & 4 & 3 & 2 & 10 & 2 & - & 5 & 3 & 1 & 1 & 1 & 1 & 3 & 3 & 3 \\
\hline Cirurgia obstétrica & 1 & 1 & 1 & 6 & 2 & - & 10 & 8 & 4 & 8 & 6 & 7 & 3 & 3 & 3 \\
\hline \multicolumn{16}{|l|}{$\begin{array}{l}\text { Cirurgia traumato- } \\
\text { ortopédica }\end{array}$} \\
\hline Cirurgia pediátrica & 4 & 4 & 4 & 4 & 1 & - & 6 & 4 & 2 & 6 & 5 & 3 & 4 & 4 & 4 \\
\hline Intensificador & & & & & & & & & & & & & & & \\
\hline de imagens & - & - & - & 9 & 3 & - & 2 & 2 & 1 & 5 & 5 & 5 & 4 & 4 & 4 \\
\hline
\end{tabular}

${ }^{a}$ D: disponibilidade (o hospital possui o recurso); A: adequação (o recurso está disponível em quantidade adequada); S: suficiência (o recurso está disponível em quantidade suficiente). 
Nove hospitais analisados no Distrito Federal dispunham de anestesiologia, tendo o serviço sido considerado adequado em seis unidades e suficiente em apenas dois. Ecocardiografia, cirurgia pediátrica e cirurgia traumato-ortopédica eram realizadas em apenas seis hospitais e foram consideradas suficientes em três, dois e um serviço, respectivamente. Cirurgia cardiovascular e neurocirúrgica estavam disponíveis em apenas cinco hospitais, um terço do que seria adequado. É importante assinalar que cirurgias traumato-ortopédicas são oferecidas em menos da metade dos hospitais.

No Rio de Janeiro, dos 16 hospitais analisados, apenas nove realizavam neurocirurgia, e em apenas três deles o serviço foi considerado suficiente. Merece destaque o fato de que apenas um dos hospitais realizava cirurgia cardiovascular. Um hospital possuía banco de tecidos, que foi considerado adequado e suficiente.

Em Curitiba, as instituições hospitalares possuíam recursos tecnológicos para todos os procedimentos investigados. Na maioria dos casos, os procedimentos eram realizados de forma adequada e suficiente.

Para avaliar a estrutura e apreender de forma conjunta a adequação e a suficiência dos recursos tecnológicos hospitalares, flexibilizamos o indicador "proporção de hospitais com recursos tecnológicos (mínimos e indispensáveis) adequados e suficientes". Desta forma, para serem considerados adequados, os hospitais deveriam ter pelo menos dois dos seguintes itens: análise clínica/laboratorial, eletrocardiografia, radiologia convencional, endoscopia, ultra-sonografia e banco de sangue. Contudo, segundo a avaliação dos gestores, uma unidade poderia oferecer eletrocardiografia de forma adequada mas não necessariamente suficiente, face à grande demanda de pacientes.

Nessa análise, Curitiba apresentou o melhor resultado, pois $100 \%$ dos hospitais possuíam recursos tecnológicos (mínimos e indispensáveis) adequados e suficientes; em seguida veio Manaus, com $85,7 \%$, e Rio de Janeiro, com 66,7\%. No Distrito Federal, apenas $33,3 \%$ dos hospitais apresentavam pelo menos dois dos seis itens tecnológicos indispensáveis em condições adequadas e suficientes. Recife apresentou o pior resultado em relação aos recursos tecnológicos, uma vez que nenhum dos hospitais atendeu os requisitos propostos no indicador.

Outro indicador utilizado foi a "proporção de hospitais de referência para $\mathrm{o}$ atendimento às vítimas de acidentes e violências com número mínimo de profissionais indispensáveis para seu funcionamento". De acordo com o Ministério da Saúde, os profissionais indispensáveis nos hospitais de referência são: cirurgião geral, cirurgião cardiovascular, cirurgião pediátrico, pediatra, clínico geral, anestesiologista, cardiologista, angiologista, enfermeira, intensivista, responsável técnico, técnico/auxiliar de enfermagem, cirurgião vascular, cirurgião bucomaxilofacial, neurocirurgião, neuropediatra, hematologista, assistente social, psicólogo, nutricionista, fisioterapeuta, terapeuta ocupacional e farmacêutico. A exigência mínima é que o hospital tenha 15 dos 23 profissionais considerados imprescindíveis para esse nível de atenção.

Em Curitiba, 100\% dos hospitais atenderam ao critério estabelecido; em Manaus, 85,7\%; em Recife e no Rio de Janeiro, 50\%; e em Brasília, apenas $36,4 \%$ dos hospitais analisados apresentavam o número mínimo de profissionais indispensáveis para o funcionamento de hospitais de referência.

\section{Transporte de pacientes, suporte de laboratório, articulação com a central de regulação, rotinas e protocolos e oferta de reabilitação}

Em todas as quatro capitais e Distrito Federal, a maioria dos serviços possuía mecanismos próprios de articulação com outros serviços para a realização de transporte e transferência de pacientes e para suporte ininterrupto de laboratório de radiologia e de patologia clínica. Além disso, a maioria das unidades estava articulada à central de regulação ou à rede pactuada do município. Quando não possuíam mecanismos próprios para o cumprimento des- sas ações, os serviços se associavam a outros para cumpri-los.

Em Manaus, apenas um hospital não possuía mecanismo próprio para a transferência e transporte de pacientes. Em Recife, apenas um serviço não estava articulado à central de regulação ou à rede pactuada. No Distrito Federal, quatro hospitais não dispunham de mecanismos próprios para suporte ininterrupto de radiologia e seis não possuíam laboratório de patologia clínica. Além disso, dois hospitais não estavam articulados à central de regulação. No Rio de Janeiro, três hospitais não apresentavam mecanismos próprios para o transporte de pacientes, um não possuía mecanismos próprios para suporte ininterrupto de radiologia, e quatro não dispunham desses mecanismos para laboratório de patologia clínica. Além disso, quatro não estavam articulados à rede pactuada do município. Em Curitiba, apenas um hospital não possuía mecanismo próprio para suporte ininterrupto de radiologia e, para isso, associava-se a outros.

Os indicadores de organização dos serviços permitiram comparar a situação das cidades estudadas. Em Manaus, Recife, Distrito Federal e Curitiba, $100 \%$ dos hospitais possuíam mecanismos próprios ou articulados com outros serviços para a realização de transferência e transporte de pacientes. $\mathrm{O}$ Rio de Janeiro se diferenciou, com uma proporção de 91,7\%, pois um dos hospitais não possuía mecanismo próprio nem estava articulado com outros. Esses resultados se repetiram para o indicador que avaliava a proporção de unidades com mecanismos próprios ou articulados para suporte ininterrupto de laboratório de radiologia e patologia clínica $(100 \%$ em Manaus, Recife, Distrito Federal e Curitiba; $91,7 \%$ no Rio de Janeiro).

A informação a respeito da articulação com a central de regulação merece maior aprofundamento, pois em todas as cidades, os gestores e profissionais entrevistados relataram dificuldades na interação entre os diferentes serviços e entre os setores do mesmo hospital. No Rio de Janeiro, não há uma central de regulação de leitos implantada. Durante algum 
tempo, havia uma estratégia informal entre as emergências hospitalares e o serviço pré-hospitalar móvel, baseada na comunicação por meio de rádio e coordenada por uma médica da Secretaria Municipal de Saúde. Por outro lado, em todas as capitais investigadas, a maioria dos gestores hospitalares mencionou dispor de rotinas e protocolos para atenção às vítimas de acidentes e violências, embora tal relato esteja em contradição com a afirmação dos gestores de não conhecerem a PNRMAV (4).

A "proporção de unidades em que os gestores dizem ter rotinas e protocolos de atendimento às vítimas de acidentes e violência acessíveis aos profissionais" constitui um indicador indireto da qualidade da atenção hospitalar, pois a existência de normas para atendimento às vítimas de acidentes e violências orienta, racionaliza e reduz os custos dos serviços. O Rio de Janeiro apresentou o pior resultado para esse indicador: somente $68,8 \%$ $(n=11)$ dos hospitais investigados na cidade disponibilizavam essas rotinas e protocolos para os profissionais. Em Manaus, $83,3 \%(n=5)$ possuíam esses dispositivos; em Brasília, 86,7\% ( $n=$ 13); Recife e Curitiba obtiveram o melhor resultado: $100 \%(n=10)$ dos serviços hospitalares investigados nessas duas capitais disponibilizavam rotinas e protocolos de atenção às vítimas de acidentes e violências para os profissionais.

TABELA 3. Distribuição dos serviços hospitalares segundo a oferta de reabilitação, Brasil, 2006

\begin{tabular}{lccccc}
\hline \multicolumn{1}{c}{ Ações realizadas } & $\begin{array}{c}\text { Manaus } \\
(n=7)\end{array}$ & $\begin{array}{c}\text { Recife } \\
(n=10)\end{array}$ & $\begin{array}{c}\text { Distrito Federal } \\
(n=15)\end{array}$ & $\begin{array}{c}\text { Rio de Janeiro } \\
(n=16)\end{array}$ & $\begin{array}{c}\text { Curitiba } \\
(n=5)\end{array}$ \\
\hline $\begin{array}{l}\text { Reabilitação } \\
\begin{array}{l}\text { Suporte e acompanhamento } \\
\text { às vítimas }\end{array}\end{array}$ & 6 & 4 & 7 & 7 & 2 \\
$\begin{array}{l}\text { Suporte e acompanhamento } \\
\text { às famílias das vítimas }\end{array}$ & 3 & 4 & 6 & 12 & 4 \\
$\begin{array}{l}\text { Suporte e acompanhamento } \\
\text { aos praticantes de acidentes } \\
\text { e violências }\end{array}$ & - & 5 & 3 & 9 & 3 \\
$\begin{array}{l}\text { Suporte e acompanhamento } \\
\text { às equipes que realizam o } \\
\text { atendimento }\end{array}$ & 4 & 5 & 4 & 2 & - \\
\hline
\end{tabular}

Em relação à "oferta de reabilitação", além de insuficiente, ela é limitada a algumas atividades voltadas para o tratamento de fisioterapia, sem levar em consideração o acompanhamento dos familiares, das vítimas de acidentes e violências, dos agentes agressores e toda a parte de reinserção social que esses pacientes necessitam. Mesmo assim, poucos hospitais dispunham de profissionais de saúde para essa atividade. Os resultados mais freqüentes foram relacionados ao suporte e ao acompanhamento das vítimas (tabela 3).

\section{Resultados hospitalares}

Por fim, trabalhamos com um indicador fundamental para a avaliação de resultados da atenção hospitalar às vítimas de acidentes e violências, a saber, a taxa de mortalidade por causas externas nas emergências. Infelizmente, poucos serviços dispunham dessa informação de forma confiável. Os dados coletados apresentaram uma variabilidade de 0,10 a $51 \%$ entre as localidades estudadas, portanto não podem ser considerados fidedignos.

\section{Síntese dos casos analisados segundo a ótica dos gestores}

Manaus centraliza os serviços de saúde de maior complexidade do 
armas brancas e afogamentos) e, sobretudo, o difícil acesso da população ribeirinha aos serviços. Agravando esse cenário, constata-se que a municipalização em todo o Estado do Amazonas é incipiente, provocando a concentração da demanda e dos serviços em Manaus. Os relatos revelam a falta de interlocução entre os setores, inclusive com os serviços federais que compõem a rede, e a falta de intersetorialidade no atendimento, aspectos considerados muito relevantes pela PNRMAV (4).

Na Cidade de Recife foram investigados 10 hospitais, todos estaduais, sendo que dois oferecem mais de um serviço de atenção às vítimas de acidentes e violências. Dentre os aspectos estudados, ganham relevo o déficit de leitos de emergência e de UTI, a superlotação dos hospitais e das emergências, a sobrecarga de demanda de atendimento a pacientes da região metropolitana, o número insuficiente de profissionais e a escassez de serviços de traumato-ortopedia, disponíveis em poucos hospitais.

O registro deficitário das informações sobre atendimentos, a descontinuidade do recebimento de medicamentos, a precariedade da referência e contra-referência e a ausência de ações de prevenção e de promoção da saúde na esfera hospitalar também constituem características problemáticas relevantes apontadas pelos gestores, mas comuns às demais capitais analisadas.

No Distrito Federal, 15 hospitais próprios compõem a rede de atenção às vítimas de acidentes e violências, sendo 13 de gestão estadual e dois vinculados ao governo federal. A maior dificuldade referida pelos gestores é a ausência de recursos destinados aos leitos de UTI e UTSI, uma vez que os leitos existentes não suprem as necessidades da demanda.

Na rede hospitalar, $80 \%$ dos serviços estão articulados através da central de regulação ou por meio da rede pactuada; $80 \%$ também dispõem de recursos tecnológicos essenciais ao atendimento. São eles: anestesiologia, cirurgia obstétrica, banco de sangue, eletrocardiograma, radiologia convencional, ultra-sonografia e análise clínica/laboratorial. Esses serviços foram considerados pelos gestores como adequados, porém insuficientes. Os profissionais entrevistados no Distrito Federal fizeram uma avaliação mais crítica do que os gestores de outras cidades e reconheceram que os serviços hospitalares apresentam um nível de qualidade de atendimento mediano e insatisfatório, necessitando ser ainda muito melhorado.

No Rio de Janeiro, dos 16 hospitais que compõem a rede de atenção às vítimas de acidentes e violências, sete pertencem à esfera municipal, cinco ao estado, e quatro são federais. Essa rede se caracteriza pela presença de serviços de referência com alta qualificação técnica, mas que, à época da pesquisa, encontrava-se desorganizada, fragmentada e desarticulada, problema superado após a instalação de uma central de encaminhamentos. As entrevistas indicaram que o atendimento pré-hospitalar não estava articulado com a rede hospitalar, gerando um tempo médio de atendimento de 66 minutos, muito acima da recomendação internacional (19). Segundo os gestores, apesar de os hospitais possuírem serviços e recursos humanos qualificados, seu número é insuficiente para atender à demanda. Os profissionais assinalaram também falhas na informação, falta de insumos e de equipamentos e precariedade do serviço de manutenção.

Em Curitiba, dos cinco hospitais que atendem vítimas de acidentes e violências, três são conveniados com o SUS; um hospital é estadual e um é federal. Todos estão articulados com a central de regulação, e a estrutura para atendimento é considerada adequada, porém insuficiente nos períodos em que ocorre aumento da demanda. Em determinados horários do dia e em datas especiais, sobretudo quando há eventos associados a um elevado consumo de álcool, incrementam-se os acidentes e as violências, e a rede hospitalar para urgências e emergências não consegue absorver toda a demanda.

Assim como observado em outras capitais, a menção à carência de leitos de UTI foi particularmente freqüente entre os gestores e profissionais dos hospitais de Curitiba.

\section{DISCUSSÃO}

A PNRMAV estabelece uma série de diretrizes e princípios cujo objetivo primordial é a busca de uma atenção qualificada para a população vítima de acidentes e violências nos vários níveis de atenção do sistema de saúde.

No que concerne à atenção de urgência, é importante reconhecer que os problemas apresentados sobre o atendimento às vítimas de acidentes e violências se relacionam com e também se agravam diante da permanência histórica de problemas estruturais resultantes do pouco investimento na esfera da atenção básica. A isso se alia uma cultura popular que busca uma atenção imediata e resolutiva para seus males, associada a uma interpretação própria, por parte da população usuária, do que seja uma situação de emergência, levando à utilização indevida dos prontos-socorros e dos hospitais de urgência e emergência e gerando, assim, uma considerável sobrecarga para esses serviços. Talvez este seja um dos maiores problemas hoje existentes nas emergências das capitais estudadas, independentemente das questões especificamente relacionadas a acidentes e violências, que vêm sendo amplamente investigadas $(9,20)$. Nesse sentido, destacase o estudo realizado por $\mathrm{O}^{\prime}$ Dwyer et al. (21) sobre a qualidade dos serviços de emergência no Rio de Janeiro, que demonstrou a utilização indevida desses serviços por $65 \%$ dos pacientes, os quais poderiam ter sido atendidos em ambulatórios. Desses pacientes, $36 \%$ não dão seguimento ambulatorial à patologia que motivou a procura do serviço de emergência, como indicado pelos autores, que também ressaltam que os serviços de emergência nessa capital funcionam acima de sua capacidade máxima, com taxa de ocupação de leitos acima de $100 \%$, número insuficiente de profissionais (muitos sem treinamento adequado), excesso de demanda e gerenciamento precário de recursos. Os 
autores observam ainda a falta de leitos de retaguarda.

$\mathrm{O}$ acúmulo de doentes nos serviços de emergência, que tanto preocupa no Brasil, parece ser um problema universal $(21,22)$. Uma pesquisa realizada na Jordânia com 2841 pacientes classificou como atendimento de urgência e emergência apenas 9\% do total atendido (23). Na Suécia, a proporção de atendimentos inapropriados na emergência varia de 30 a 50\%, apesar da expansão da atenção básica, como referem Kakrau e Hassler (24). Nos Estados Unidos, esse tem sido um tema recorrente de pesquisa e de atenção por parte de gestores e planejadores do setor (25).

Outro problema relevante freqüentemente apontado e também mencionado pelos gestores entrevistados nesta pesquisa, sobretudo quanto ao atendimento de violências e acidentes, é a falta de investimento financeiro na capacitação de pessoal, conforme já assinalado por O'Dwyer et al. (21), dificultando a implantação da política em sua integralidade. Além desses impedimentos, os profissionais entrevistados ressaltaram a questão da integração de várias instâncias para a efetiva implementação da PNRMAV, um grande desafio que exige ações interdisciplinares, multiprofissionais e multissetoriais.

Neste artigo, foram apresentadas informações importantes para a melhor adequação dos serviços de emergência oferecidos pelo SUS no sentido de atender o quadro de morbimortalidade por violências e acidentes. Os dados apontam para alguns aspectos: a importância do fortalecimento da rede e da articulação entre os serviços; a relevância do investimento tanto em equipamentos como em sua manutenção no ritmo exigido pelos atendimentos; e a importância da formação específica e permanente dos profissionais que atuam nesse setor especializado. Em relação à organização da rede hospitalar, ressaltam-se alguns pontos positivos encontrados nas cidades estudadas: a maioria dos hospitais possui mecanismos próprios de articulação com outros serviços para a realização de transporte e transferência de pacientes, para suporte ininterrupto de laboratório de radiologia e de patologia clínica, além de estarem articulados a central de regulação ou rede municipal pactuada de atendimento de urgência e emergência.

Considerando que os condicionamentos citados influenciam as condições organizacionais de assistência (22), procedeu-se a uma análise detalhada em relação às causas externas nas cinco localidades. Chama atenção o fato de que a deficiência de infraestrutura das urgências hospitalares e os problemas organizacionais são bastante diferentes nas cinco localidades, embora existam problemas comuns. Destaca-se, como ponto comum, a insuficiência de leitos de emergência e de UTI, a insuficiência de recursos, a pouca oferta de exames (por exemplo, tomografias), a ausência de banco de tecidos e a deficiência na oferta de neurocirurgia, cirurgia cardiovascular e cirurgia pediátrica.

No Rio de Janeiro, a rede apresenta serviços com alta qualificação técnica; no entanto, na época em que a pesquisa foi realizada, havia uma visível desarticulação das várias instâncias, fato constatado também por Azevedo et al. (26) e O'Dwyer et al. (21). Também faltam a essa rede insumos, equipamentos e sua manutenção de forma e em tempo adequados. Em Curitiba, os hospitais estão conectados à central de regulação, e a estrutura para atendimento é considerada adequada, porém os gestores mencionam limitações do sistema em determinados horários do dia e em datas festivas que usualmente concentram maiores ocorrências.

A articulação entre os serviços de referência e contra-referência é um problema em todas as capitais. Estudos sugerem que a configuração de uma rede assistencial cooperativa, envolvendo referências municipais e regionais e com fluxo regulado e apoiado pelo Serviço de Atendimento Móvel de Urgência (SAMU), amplamente difundido junto à população, melhoraria a resolutividade da atenção à saúde, reduziria a demanda às portas hospitalares de urgência e humanizaria e qualificaria o atendimento $(2,27,28)$.
Ainda, chama atenção a insuficiência do registro da taxa de mortalidade devido a causas externas nas emergências, informação estratégica para o dimensionamento da gravidade das lesões, bem como da qualidade da assistência prestada.

Finalmente, a disponibilidade e a composição das equipes nos serviços estão muito longe de cumprir o que é preconizado pelo Ministério da Saúde (exceto em Curitiba). Merecem destaque as questões institucionais que impedem a mudança, como a mentalidade de se resolver as deficiências do sistema pelo setor de urgências (26, 29). Também é claro que existe escassez de recursos financeiros e humanos, embora, por outro lado, uma cultura individualista persista entre os profissionais, dificultando a formação de grupos. No entanto, o caso de Curitiba, que, em quase todos os aspectos, é bastante exemplar, mostra que é possível conseguir uma organização coesa e racional, mesmo enfrentando os vários problemas de escassez e de financiamento do SUS.

Nos hospitais, a capacitação para o atendimento e o adequado registro das violências e acidentes são processos muito dispersos. Observa-se grande resistência dos gestores para a liberação de seu pessoal, pois isso implicaria uma queda de produtividade no montante de atendimentos. No entanto, esse é um ponto central na avaliação da qualidade do sistema (21, 22).

O não-reconhecimento da urgência como área específica de conhecimento e especialidade pode ser um dos fatores responsáveis pela falta de capacitação e de educação continuada dos profissionais, resultado esse encontrado nos hospitais avaliados na pesquisa que deu origem a este artigo (5). As universidades e alguns serviços têm incorporado estratégias didáticas e assistenciais do modelo anglo-saxão por meio da oferta de cursos específicos, como Suporte Avançado de Vida no Trauma (Advanced Life Trauma Support, ATLS). Todavia, essas iniciativas, sem continuidade através de uma proposta de educação permanente, não garantem 
o aperfeiçoamento dos serviços e das pessoas (19).

Em resumo, a pesquisa mostra que há dificuldades comuns e diferentes em cada localidade analisada, e que há ainda um longo caminho a ser percorrido no processo de institucionalização da PNRMAV (4), até pelo fato de essa política ser ainda bastante recente. Por outro lado, êxitos obtidos violence in Brasil. Cienc Saude Coletiva. 2006;11(2):363-74.

2. Santos JS. Da fundação do Hospital das Clínicas à criação da unidade de emergência e sua transformação em modelo nacional de atenção às urgências. Medicina. 2002;35(3): 403-18.

3. Brasil, Ministério da Saúde. Portaria $n^{\circ} 737$ MS/GM, de 16 de maio de 2001. Política Nacional de Redução da Morbimortalidade por Acidentes e Violências. Brasília: Ministério da Saúde; 2001. Disponível em: http://www. saude.ba.gov.br/samu/arquivos/por tarias/Portaria\%20n\%C2\%BA\%20737\%20G M\%2016\%20de\%20maio\%20de\%202001.doc. Acessado em 14 de dezembro de 2007.

4. Brasil, Ministério da Saúde. Política Nacional de Redução de Morbimortalidade por Acidentes e Violência (PNRMAV). Brasília: Ministério da Saúde; 2002.

5. Minayo MCS, Deslandes SF, orgs. Análise diagnóstica da política nacional de saúde para redução de acidentes e violências. Rio de Janeiro: Fiocruz; 2007.

6. Brasil, Ministério da Saúde. Portaria $\mathrm{n}^{\circ}$ $814 / \mathrm{GM}$, de $1^{\circ}$ de junho de 2001. Estabelece o conceito geral, os princípios e as diretrizes da regulação médica das urgências. Brasília: Ministério da Saúde; 2001. Disponível em: http://www.mp.ba.gov.br/atuacao/cidada nia/gesau/legislacao/temas/urgencia/ portaria_GM_814.pdf. Acessado em 14 de dezembro de 2007.

7. Brasil, Ministério da Saúde. Portaria $n^{\circ}$ 824/GM, de 24 de junho de 1999. Aprova o texto de normatização de atendimento préhospitalar, constante do anexo desta portaria. Brasília: Ministério da Saúde; 1999. Disponível em: http://www.mp.ba.gov.br/ atuacao/cidadania/legislacao/saude/sus/ portaria_824_99.asp. Acessado em 14 de dezembro de 2007.

8. Brasil, Ministério da Saúde. Portaria $n^{\circ} 2.048$, de 5 de novembro de 2002. Aprova o regulamento técnico dos sistemas estaduais de urgência e emergência. Atendimento pré-hospitalar. Brasília: Ministério da Saúde; 2002. Disponível em: http://www.saude.mg.gov. br/atos_normativos/legislacao-sanitaria/ estabelecimentos-de-saude/urgencia-eemergencia/portaria_2048_B.pdf. Acessado em 14 de dezembro de 2007. em alguns aspectos específicos em cada uma das capitais estudadas indicam que, embora complexo, o processo de implantação da política vem ocorrendo e pode acontecer com muito mais adequação se houver investimentos nos pontos críticos, relativos à infra-estrutura, à articulação das redes, à organização dos serviços e à formação dos profissionais. A cultura organizacional precisa ser um ponto de inflexão.

Termina-se reafirmando que os sucessos particulares aqui ressaltados provam que é possível incluir o tema da violência e dos acidentes na pauta da saúde pública e que é possível atuar, a partir do setor e com as estratégias que lhe são peculiares em diferentes níveis, a favor da vida dos brasileiros.

\section{REFERÊNCIAS}

9. Deslandes SF. Frágeis deuses: profissionais da emergência entre os danos da violência e a recriação da vida. Rio de Janeiro: Fiocruz; 2002.

10. Brasil, Ministério da Saúde. Impacto da violência na saúde dos brasileiros. Brasília: Ministério da Saúde; 2005.

11. Denzin NK. The research act. Chicago: Aldine; 1973.

12. Minayo MC, Sanches O. Quantitativoqualitativo: oposição ou complementaridade? Cad Saude Publica. 1993;9(3):239-62.

13. Minayo MCS, Assis SG, Souza ER. Avaliação por triangulação de métodos. Rio de Janeiro: Fiocruz; 2005.

14. Brasil, Ministério da Saúde. Portaria $n^{\circ}$ 1.863/GM, de 29 de setembro de 2003. Institui a política nacional de atenção às urgências, a ser implantada em todas as unidades federadas, respeitadas as competências das três esferas de gestão. Brasília: Ministério da Saúde; 2003. Disponível em: www.saude.ba. gov.br/samu/arquivos/portarias/portaria 1863 gm.doc. Acessado em 14 de dezembro de 2007.

15. Brasil, Secretaria de Vigilância Sanitária. Portaria n⿳0 466/SVS, de 4 de junho de 1998. Estabelece o regulamento técnico para o funcionamento dos serviços de tratamento intensivo. Brasília: Ministério da Saúde; 1998. Disponível em: http://www.ufpe.br/utihc/ portaria466.htm. Acessado em 14 de dezembro de 2007.

16. Brasil, Ministério da Saúde. Portaria $n^{\circ}$ 479/GM, de 15 de abril de 1999. Cria mecanismos para a implantação dos sistemas estaduais de referência hospitalar em atendimento de urgências e emergências. Brasília: Ministério da Saúde; 1999. Disponível em: http: / / dtr2001.saude.gov.br/sas / POR TARIAS/Port99/GM/GM-0479.html. Acessado em 14 de dezembro de 2007.

17. Lauritsen JM, ed. EpiData Data Entry — Data management and basic statistical analysis system. Odense Denmark: EpiData Association; 2000-2008. Disponível em: http:// www.epidata.dk. Acessado em 14 de dezembro de 2007.

18. Brasil, Ministério da Saúde; 2007. Plano nacional de atenção às urgências. Brasília: Ministério da Saúde; 2004

19. Malvestio MAA, Souza RMC. Suporte avançado à vida: atendimento a vítimas de acidentes de trânsito. Rev Saude Publica. 2002;36(5):584-9.

20. Jacquemot A. Qu'est-ce qu'une urgence? Quelques réflexions sur les définitions biomédicales de l'urgence médicale. Em: Bernabé J, Confiant R. Au visiteur lumineux Des îles créoles aux sociétés plurielles. PetitBourg: Ibis Rouge Editions; 2000. Pp. 575-86.

21. O'Dwyer G, Oliveira SP, De Seta MH. Avaliação dos serviços hospitalares de emergência do programa Qualisus no Rio de Janeiro. Rev C S Col. Disponível em: http://www. cienciaesaudecoletiva.com.br. Acessado em 22 de junho de 2008.

22. Donabedian A. The quality of care; how can it be assessed? JAMA. 1988;260(12):1743-8.

23. Abdallat AM, Al-Smadi I, Abbadi MD. Who uses the emergency room services? East Mediterr Health J. 2000;6(5):1126-9.

24. Krakau I, Hassler E. Provision for clinic patients in the ED produces more nonemergency visits. Am J Emerg Med. 1999;17(1): 18-20.

25. Coleman P, Irons R, Nicholl J. Will alternative immediate care services reduce demands for non-urgent treatment at accident and emergency? Emerg Med J. 2001;18(4):482-7.

26. Azevedo CS, Fernandes MIA, Carreteiro TC. Sob o domínio da urgência: a prática de diretores de hospitais públicos do Rio de Janeiro, Brasil. Cad Saude Publica. 2007;23(10): 2410-20.

27. Derlet RW, Wishio D, Cole LM, Silva Júnior J. Triage of patients out of emergency department: three-year experience. Am J Emerg Med. 1992;10(3):195-9.

28. Labarere J, Torres JP, Fourny M, Argente P, Gfensburger X, Menthonnex P. Patient compliance with medical advice given by telephone. Am J Emerg Med. 2003;21(4):288-92.

29. Mesquita Filho M, Mello MHP. Características da morbidade por causas externas em serviços de urgência. Rev Bras Epidemiol. 2007;10(4):579-91.

Manuscrito recebido em 7 de abril de 2008. Aceito em versão revisada em 31 de julho de 2008. 
ABSTRACT Objective. To analyze positive results, challenges, limitations, and potential benefits associated with the Brazilian Policy for the Reduction of Morbidity and Mortality due to Accidents and Violence (PNRMAV) with regard to hospital emergency and urgent

Emergency care for victims of accidents and violence in Brazil

Methods. From 2004-2007, a study was carried out in four Brazilian state capitals and the Federal District. The following areas were evaluated: the availability of standard practices for managing victims of accident or violence; availability and quality of adult and pediatric intensive care beds; patient management training for health care professionals; communication among the health care areas to provide better care to victims; and structure and organization of hospital services. The study included an exploratory stage, fieldwork, analysis, and dissemination of data. Both quantitative (absolute and relative frequencies) and qualitative methods (analysis of interactions between explicit and inferred information from statements and field records) were employed in the analysis.

Results. The study revealed positive results from the implementation of PNRMAV. The following focal areas were identified: (1) the need to invest in the response capacity of primary care services, (2) the importance of strengthening and promoting the emergency and urgent care network, (3) the importance of investing in appropriate equipment and of planning maintenance so that availability is not impacted, and (4) the need to invest in continuing education for health care professionals.

Conclusions. The partial success obtained with PNRMAV shows that it is possible to include the topic of violence and accidents in the public health agenda and to implement life-promoting strategies at different levels.

Key words Accidents, emergency medicine, health public policy, violence, Brazil. 